\title{
Spacelike Hypersurfaces with Prescribed Boundary Values and Mean Curvature
}

\author{
Robert Bartnik ${ }^{1}$ and Leon Simon ${ }^{2}$ \\ 1 Department of Mathematics, Princeton University, Princeton, NJ 08544, USA \\ 2 Department of Mathematics, Australian National University, Canberra, A.C.T. 2600, Australia
}

\begin{abstract}
We consider the boundary-value problem for the mean curvature operator in Minkowski space, and give necessary and sufficient conditions for the existence of smooth strictly spacelike solutions. Our main results hold for non-constant mean curvature, and make no assumptions about the smoothness of the boundary or boundary data.
\end{abstract}

\section{Introduction}

An important problem in classical Relativity is that of determining existence and regularity properties of maximal and constant mean curvature hypersurfaces. These are spacelike submanifolds of codimension one in the spacetime manifold, with the property that the trace of the extrinsic curvature is respectively zero, constant. Such surfaces are important because they provide Riemannian submanifolds with properties which reflect those of the spacetime. For example, if the weak energy condition is satisfied, then a maximal hypersurface has positive scalar curvature. This fact was important in the initial proof of the positive mass conjecture [SY]. Other applications can be found in [ES] and [MT].

However, the use of these surfaces is restricted presently because their analytical properties are not well understood. By considering extrema of the associated variational problem, natural conditions for the existence of weak solutions can readily be established [Av]. These extrema are a priori only Lipschitz-continuous and may be lightlike ("go null", in the terminology of [MT]). Smoothness will follow from non-linear elliptic theory, provided we can show that they do not go null.

We consider this problem in flat Minkowski space $\mathbb{L}^{n+1}$, and give necessary and sufficient conditions to ensure that extrema of the variational problem do not go null. To be precise, we show (in Theorem 4.1) that for a given bounded domain $\Omega \subset \mathbb{R}^{n}$ there is a smooth strictly spacelike solution of the Lorentz mean curvature equation with specified boundary values on $\partial \Omega$, provided only that the given boundary data spans some spacelike hypersurface, and the mean curvature function is smooth and bounded on $\Omega \times \mathbb{R}$. Since this result makes no assumptions about the regularity of the boundary data or boundary, it can be applied to problems over unbounded domains. We illustrate this in Sect. 4, using a barrier construction due to Treibergs [T].

We mention that some results for the Dirichlet problem for zero mean curvature were obtained in $[\mathrm{B}, \mathrm{F}]$; however both these authors make very 
restrictive assumptions about the boundary data. Using different techniques to those presented here, Cheng and Yau proved a Bernstein theorem for maximal hypersurfaces, extending earlier work of Calabi [CE, CY].

The outline of the present paper is as follows: In Sect. 1 we gather some simple results about the variational problem in flat space. In Sect. 2 we prove a "mean value" inequality and the a priori estimate (2.22). This estimate plays a key role in controlling the variational extremum. The Dirichlet problem for smooth domains and data is solved in Sect. 3, using the cone-like barrier functions (3.2) and a global a priori gradient estimate. The "anti-peeling" Theorem 3.2 is perhaps of independent interest. It says roughly that if the extremal surface contains a piece of light ray, then the surface cannot "peel away" from the extended light ray. Finally, in Sect. 4 we collect these results to prove the interior regularity of the variational extremal.

"Postscript: C. Gerhardt has independently obtained the gradient estimate of Theorem 3.5, and has shown that it can be generalized to non-flat spacetimes."

\section{The Variational Problem}

We denote Minkowski space by $\mathbb{L}^{n+1}=\left\{(x, t): x \in \mathbb{R}^{n}, t \in \mathbb{R}\right\}$, with the flat metric $\sum_{i=1}^{n} d x_{i}^{2}-d t^{2}$. Recall that $C^{0,1}(\Omega)$ is the class of locally Lipschitz functions on $\Omega$. For this and other notation, we refer to [GT].

Let $M$ be an $n$-dimensional hypersurface in $\mathbb{L}^{n+1}$ which can be represented as the graph of $u \in C^{0,1}(\Omega)$, where $\Omega$ is a bounded domain in $\mathbb{R}^{n}$. We will say that $M$ is

weakly spacelike if $|D u| \leqq 1$ a.e. in $\Omega$,

spacelike if $|u(x)-u(y)|<|x-y|$ whenever $x, y \in \Omega, x \neq y$

and the line segment $\overline{x y} \subset \Omega$, and

strictly spacelike if $M$ is spacelike, $u \in C^{1}(\Omega)$ and $|D u|<1$ in $\Omega$.

Similar terminology will be used to describe the function $u$. Note that $M$ must be strictly spacelike for the Lorentz mean curvature to be well defined, but weakly spacelike is sufficient to define the area integral, $\int_{\Omega} \sqrt{1-|D u|^{2}} d x$.

For physical reasons, a line of slope 1 is called a light ray. The light cone $C_{\left(\xi, \xi_{n+1}\right)}$ at $\left(\xi, \xi_{n+1}\right) \in \mathbb{L}^{n+1}$ is the union of all light rays containing $\left(\xi, \xi_{n+1}\right)$ :

$$
C_{\left(\xi, \xi_{n+1}\right)}=\left\{\left(x, x_{n+1}\right) \in \mathbb{L}^{n+1}:\left|x_{n+1}-\xi_{n+1}\right|=|x-\xi|_{\mathbb{R}^{n}}\right\}
$$

If $C_{\xi^{\prime}} \cap M=\left\{\xi^{\prime}\right\}$ for all $\xi^{\prime} \in M$, then $M$ (and $u$ ) is said to be acausal. This says exactly that distinct points of $M$ are spacelike separated. We observe that $M$ spacelike is a weaker condition than $M$ acausal, although the two notions coincide when $\Omega$ is convex. The main theorems here do not require convexity of $\Omega$, and consider spacelike surfaces, more generally than acausal surfaces.

Unless otherwise stated, no assumptions will be made about the regularity of $\Omega$. The possible pathology of $\partial \Omega$ leads to the following 
Definition. If $\varphi: \partial \Omega \rightarrow \mathbb{R}$, and $\psi: \Omega \rightarrow \mathbb{R}$ is spacelike, then we agree that

$$
\psi=\varphi \quad \text { on } \quad \partial \Omega
$$

means that, for every $x_{0} \in \partial \Omega$, and every open straight line segment $l$ contained in $\Omega$ and with endpoint $x_{0}$,

$$
\lim _{\substack{x \rightarrow x_{0} \\ x \in l}} \psi(x)=\varphi\left(x_{0}\right) .
$$

Throughout this work, boundary values will be taken in the sense of this definition. Of course, if $\partial \Omega$ is Lipschitz, then this is the usual definition. It is not difficult to construct examples with points $x_{0} \in \partial \Omega$ at which $\psi$ is not related to $\varphi\left(x_{0}\right)$ by this definition [since there may not be any line segments $l$ as in (1.1)], but it is also not hard to show that the set of "regular" points (i.e. boundary points with such line segments) is dense in $\partial \Omega$. For later reference, we note that if $\psi_{1}$ and $\psi_{2}$ are two spacelike functions on $\Omega$, agreeing with $\varphi$ in the sense of (1.1), then $\psi_{1}-\psi_{2}$ extends to a Lipschitz function on $\mathbb{R}^{n}$ which vanishes outside $\Omega$.

The Dirichlet problem

$$
\begin{aligned}
\mathscr{M} u(x) & =\frac{1}{\sqrt{1-|D u|^{2}}} \sum_{i, j=1}^{n}\left(\delta_{i j}+\frac{D_{i} u D_{j} u}{1-|D u|^{2}}\right) D_{i j} u=H(x, u(x)), \\
u & =\varphi \text { on } \partial \Omega,
\end{aligned}
$$

where $H: \Omega \times \mathbb{R} \rightarrow \mathbb{R}$ and $\varphi: \partial \Omega \rightarrow \mathbb{R}$ are given bounded functions, is the EulerLagrange equation of the variational problem

$$
\left.\begin{array}{ll}
\text { maximize } & I(u)=\int_{\Omega}\left\{\sqrt{1-|D u|^{2}}-\int_{0}^{u(x)} H(x, t) d t\right\} d x \\
\text { amongst } \quad u \in \mathscr{C}(\varphi, \Omega)=\left\{w \in C^{0,1}(\Omega): w=\varphi \text { on } \partial \Omega \text {, and }|D w| \leqq 1 \text { a.e. in } \Omega\right\} .
\end{array}\right\}
$$

Since $\Omega$ is also bounded, $I(\cdot)$ is uniformly bounded on $\mathscr{C}(\varphi, \Omega)$. The equicontinuity of $\mathscr{C}$ then gives a uniformly convergent maximizing sequence $u_{k} \rightrightarrows u \in \mathscr{C}(\varphi, \Omega)$. Since the area integrand $\sqrt{1-|p|^{2}}$ is concave, a semi-continuity theorem of Serrin ([M] Theorem 1.8.1) shows that

$$
\limsup _{k \rightarrow \infty} \int_{\Omega} \sqrt{1-\left|D u_{k}\right|^{2}} d x \leqq \int_{\Omega} \sqrt{1-|D u|^{2}} d x,
$$

and hence $I(u)$ is maximal. Thus the variational problem (1.3) can be solved provided only that $\mathscr{C}(\varphi, \Omega)$ is non-empty. This upper semicontinuity result is well known also in the case of general spacetimes $[\mathrm{Av}]$.

Proposition 1.1. The variational problem can be solved iff $\mathscr{C}(\varphi, \Omega)$ is non-empty. The solution is unique if $H(x, t)$ is non-decreasing in $t$.

Proof. The first statement is clear from the above discussion.

Now suppose $u, w$ are two solutions of (1.3), and set $u_{t}=u+t(w-u)$. Then by the concavity of $\sqrt{1-|p|^{2}}$,

$$
\int_{\Omega} \sqrt{1-\left|D u_{t}\right|^{2}} d x \geqq(1-t) \int_{\Omega} \sqrt{1-|D u|^{2}} d x+t \int_{\Omega} \sqrt{1-|D w|^{2}} d x .
$$


Now $H$ non-decreasing implies that $\int_{0}^{s} H(x, t) d t$ is convex, and thus $I\left(u_{t}\right) \geqq(1-t) I(u)$ $+t I(w)$. Since $u$ and $w$ both maximize $I$, equality must hold, and hence it follows that

$$
\sqrt{1-\left|D u_{t}\right|^{2}}=(1-t) \sqrt{1-|D u|^{2}}+t \sqrt{1-|D w|^{2}} \quad \text { a.e. in } \Omega \text {. }
$$

This implies that $D u=D w$ a.e. in $\Omega$. Since $u=w$ on $\partial \Omega$, by a previous remark $u-w$ extends to a Lipschitz function on $\mathbb{R}^{n}$, vanishing outside $\Omega$. Since $D(u-w)=0$ a.e. in $\Omega$, we must have $u=w$, and the solution is unique.

The following two lemmas enable us to compare solutions of the Dirichlet problem and of the variational problem. The first is a general comparison principle.

Lemma 1.2. For $i=1,2$ suppose $u_{i}$ is the solution of (1.3) with bounded boundary data $\varphi_{i}$ and bounded mean curvature $H_{i}$. Suppose also that

$$
H_{1}(x) \leqq H_{2}(x) \quad \text { for a.e. } x \in \Omega \text {. }
$$

Then

$$
u_{2}(x) \leqq u_{1}(x)+\sup _{\partial \Omega}\left(\varphi_{2}-\varphi_{1}\right)
$$

where $\sup _{\partial \Omega}\left(\varphi_{2}-\varphi_{1}\right)$ is taken in the sense of (1.1).

Proof. Let $C=\sup _{\partial \Omega}\left(\varphi_{2}-\varphi_{1}\right)$, choose $\varepsilon>0$ and define $\tilde{u}_{1}=u_{1}+C+\varepsilon$ and $\Omega^{+}=\left\{x \in \Omega: u_{2}(x)>\tilde{u}_{1}(x)\right\}$. Assume $\Omega^{+}$is non-empty.

The function $\left(u_{2}-\tilde{u}_{1}\right)_{+}$vanishes on $\partial \Omega$ in the sense of $(1.1)$ and hence extends to a Lipschitz function vanishing outside $\Omega$. Clearly $u_{2}=\tilde{u}_{1}$ on $\partial \Omega^{+}$, again in the sense of (1.1). Define functionals $I_{1}, I_{2}$ by

$$
I_{i}(u)=\int_{\Omega^{+}}\left(\sqrt{1-|D u|^{2}}-u(x) H_{i}(x)\right) d x, \quad i=1,2 .
$$

Then $\tilde{u}_{1}$ maximizes $I_{1}$ with respect to $\left.\tilde{u}_{1}\right|_{\partial \Omega^{+}}$, and $u_{2}$ maximizes $I_{2}$ with the same boundary values. Then by uniqueness of the solution of this variational problem, $I_{2}\left(\tilde{u}_{1}\right)<I_{2}\left(u_{2}\right)$, and hence

$$
\begin{aligned}
\int_{\Omega^{+}} \sqrt{1-\left|D \tilde{u}_{1}\right|^{2}} d x & <\int_{\Omega^{+}}\left(\sqrt{1-\left|D u_{2}\right|^{2}}+\left(\tilde{u}_{1}-u_{2}\right) H_{2}\right) d x \\
& \leqq \int_{\Omega^{+}}\left(\sqrt{1-\left|D u_{2}\right|^{2}}+\left(\tilde{u}_{1}-u_{2}\right) H_{1}+\left(\tilde{u}_{1}-u_{2}\right)\left(H_{2}-H_{1}\right)\right) d x .
\end{aligned}
$$

Thus, since $\tilde{u}_{1}<u_{2}$ on $\Omega^{+}$by construction, and $H_{1} \leqq H_{2}$ on $\Omega$, this becomes

$$
\int_{\Omega^{+}}\left(\sqrt{1-\left|D \tilde{u}_{1}\right|^{2}}-\tilde{u}_{1} H_{1}\right) d x<\int_{\Omega^{+}}\left(\sqrt{1-\left|D u_{2}\right|^{2}}-u_{2} H_{1}\right) d x
$$

which contradicts the maximality of $I_{1}\left(\tilde{u}_{1}\right)$. Hence $\Omega^{+}$is empty and since $\varepsilon>0$ was arbitrary, the result follows.

If $H(x, t)$ is non-decreasing in $t$, then the concavity of $\sqrt{1-|p|^{2}}$ and of $-\int_{0}^{u(x)} H(x, t) d t$ implies that any solution of the Dirichlet problem is also a solution 
of the variational problem (see [GT, Sect. 10.5] for a discussion). Thus the above lemma allows us to compare solutions of the Dirichlet and variational problems.

The next result roughly says that the limit of solutions of the Dirichlet problem is a solution of a variational problem.

Lemma 1.3. Suppose there is a sequence $\left\{u_{k}\right\}_{1}^{\infty}$ in $C^{1}(\Omega) \cap W^{2,2}(\Omega)$ of strictly spacelike functions with (weak) mean curvatures $\mathscr{M} u_{k}=H_{k}, H_{k}$ measurable on $\Omega$ and $\sup _{\Omega}\left|H_{k}\right| \leqq \Lambda$, such that $\left\{u_{k}\right\}_{1}^{\infty}$ converges uniformly and $\left\{H_{k}\right\}_{1}^{\infty}$ converges weakly,

$$
\begin{array}{ccc}
u_{k} \rightrightarrows u & \text { in } & C^{0}(\bar{\Omega}), \\
H_{k} \rightarrow H & \text { in } & L^{2}(\Omega) .
\end{array}
$$

Then $u$ is weakly spacelike and, with respect to its own boundary values, solves the variational problem with mean curvature $H$.

Proof. Since each $u_{k}$ is weakly spacelike in $\Omega$ and $\left\{u_{k}\right\}$ converges uniformly to $u$, it easily follows that $u$ is weakly spacelike. Suppose $w \in C^{0,1}(\Omega)$ satisfies $w=u$ on $\partial \Omega$. Then we must show that $I(w) \leqq I(u)$, where $I$ is defined by

Define

$$
I(w)=\int_{\Omega}\left(\sqrt{1-|D w|^{2}}-w(x) H(x)\right) d x .
$$

$$
\Omega^{+}=\{x \in \Omega: w(x)>u(x)\} \quad \text { and } \quad \Omega_{k}=\left\{x \in \Omega: w(x)-2 / k>u_{k}(x)\right\} .
$$

We can assume (relabelling if necessary) $\sup _{\Omega}\left|u-u_{k}\right| \leqq 1 / k$, so that $\Omega_{k} \subset \Omega^{+}$. Clearly we also have $\Omega_{k}^{+} \rightarrow \Omega$ as $k \rightarrow \infty$ in the measure sense. By a previous remark, $u_{k}$ maximizes $I_{k}$, so

$$
\int_{\Omega_{k}}\left(\sqrt{1-|D w|^{2}}-(w-2 / k) H_{k}\right) d x \leqq \int_{\Omega_{k}}\left(\sqrt{1-\left|D u_{k}\right|^{2}}-u_{k} H_{k}\right) d x,
$$

since $w=2 / k=u_{k}$ on $\partial \Omega_{k}$. Since $\Omega_{k}$ and $H_{k}$ are bounded, this becomes

$$
\int_{\Omega_{k}}\left(\sqrt{1-|D w|^{2}}-w H_{k}\right) d x \leqq \int_{\Omega_{k}}\left(\sqrt{1-\left|D u_{k}\right|^{2}}-u_{k} H_{k}\right) d x+\varepsilon_{k},
$$

where $\varepsilon_{k} \rightarrow 0$ as $k \rightarrow \infty$. Thus, since $H_{k} \rightarrow H$ and $u_{k} \rightrightarrows u$, letting $k \rightarrow \infty$ and using the upper semicontinuity of the area integral, we have

$$
\begin{aligned}
\int_{\Omega^{+}}\left(\sqrt{1-|D w|^{2}}-w H\right) d x & \leqq \limsup _{k \rightarrow \infty} \int_{\Omega^{+}} \sqrt{1-\left|D u_{k}\right|^{2}} d x-\int_{\Omega^{+}} u H d x \\
& \leqq \int_{\Omega^{+}}\left(\sqrt{1-|D u|^{2}}-u H\right) d x .
\end{aligned}
$$

A similar argument applied to $\Omega^{-}=\{x \in \Omega ; w(x)<u(x)\}$ shows that $I(w) \leqq I(u)$, as required.

\section{Main Estimate for $|D u|$}

Throughout this section, $M$ will be a strictly spacelike, $n$-dimensional hypersurface, represented as the graph of a $C^{3}$ function $u(x)$. Unless otherwise stated, the domain of $u$ will be $\mathbb{R}^{n}$, so $u \in C^{3}\left(\mathbb{R}^{n}\right)$. Since $M$ is $C^{3}$ and spacelike, the induced 
metric is positive definite, giving $M$ a Riemannian structure. This enables us to generalize many well-known calculations from the theory of minimal surfaces in $\mathbb{R}^{n+1}$ to the present case (see for example [GT, MS]). Although formally the calculations are very similar, the estimates are quite different. For example, the projection of a vector onto the tangent space of $M$ is longer than the original vector.

We shall use the summation convention on repeated indices with the following ranges $1 \leqq i, j, \ldots \leqq n, 1 \leqq \alpha, \beta, \ldots \leqq n+1$. For $u$ only, we denote partial derivatives by subscripts, so $u_{i}=\frac{\partial u}{\partial x_{i}}, u_{i j}=\frac{\partial^{2} u}{\partial x_{i} \partial x_{j}}$ and so on.

The flat metric on $\mathbb{L}^{n+1}$ will be denoted $\langle\cdot, \cdot\rangle$ or $\eta=\operatorname{diag}(1,1, \ldots, 1,-1)$, so $\eta_{\alpha \beta}=\left\langle e_{\alpha}, e_{\beta}\right\rangle$ in terms of the natural basis $\left\{e_{\alpha}\right\}$ of $\mathbb{L}^{n+1}$. The norm on spacelike vectors is $\|X\|=\langle X, X\rangle^{1 / 2}$, while $|x|=\left(\sum_{1}^{n} x_{i}^{2}\right)^{1 / 2}$ denotes length in $\mathbb{R}^{n}$, and $\nabla$ is the Levi-Civita connection of $\eta$.

Since $M$ is a graph, $M=\left\{(x, u(x)): x \in \mathbb{R}^{n}, u \in C^{3}\left(\mathbb{R}^{n}\right)\right\}$, we have coordinates $\left(x_{1}, \ldots, x_{n}\right)$ on $M$ with coordinate tangent vectors $X_{i}=e_{i}+u_{i} e_{n+1}, i=1, \ldots, n$. The induced metric on $M$ is then

$$
g_{i j}=\left\langle X_{i}, X_{j}\right\rangle=\delta_{i j}-u_{i} u_{j}, \quad 1 \leqq i, j \leqq n,
$$

and hence $\operatorname{det} g_{i j}=1-|D u|^{2}$. The quantity $v=\sqrt{1-|D u|^{2}}$ will prove to be very important. Let $v$ be the upward normal to $M$, normalized by $\langle v, v\rangle=-1$,

$$
\begin{aligned}
v & =\frac{1}{v}(D u, 1)=v_{i} e_{i}+v_{n+1} e_{n+1}, \\
v_{i} & =\frac{1}{v} u_{i}, \quad i=1, \ldots, n, \quad v_{n+1}=\frac{1}{v} .
\end{aligned}
$$

The inverse $g^{i j}$ is then

$$
g^{i j}=\delta_{i j}+v_{i} v_{j}, \quad 1 \leqq i, j \leqq n .
$$

We will use raised indices only for $g^{i j}$, and nowhere else.

The second fundamental form can be calculated directly,

$$
\begin{gathered}
A_{i j}=\left\langle X_{i}, \nabla_{X_{j}} v\right\rangle=\frac{1}{v} u_{i j}, \\
\|A\|^{2}=g^{i j} g^{k l} A_{i k} A_{j l}=\frac{1}{v^{2}} g^{i j} g^{k l} u_{i k} u_{j l},
\end{gathered}
$$

and the mean curvature of $M$ is thus

$$
H=g^{i j} A_{i j}=\frac{1}{v} g^{i j} u_{i j} .
$$

Let $\mathscr{U} \subset \mathbb{L}^{n+1}$ be an open neighbourhood of $M$, and let $Y$ be a $C^{1}$ vectorfield on $\mathscr{U}$ and $f \in C^{2}(\mathscr{U})$ be such that $D_{n+1} f=0$. Then the differential operators $\delta=\operatorname{grad}_{M}$, 
$\operatorname{div}_{M}$, and $\Delta_{M}$ may be expressed

$$
\begin{aligned}
\delta f & =\delta_{\alpha} f \quad e_{\alpha}, \\
\delta_{i} f & =g^{i j} \frac{\partial}{\partial x_{j}} f, \quad i=1, \ldots, n, \quad \delta_{n+1} f=\frac{1}{v} v_{i} \frac{\partial}{\partial x_{i}} f, \\
\operatorname{div}_{M} Y & =g^{i j}\left\langle X_{i}, \nabla_{X_{j}} Y\right\rangle, \\
\Delta_{M} f & =\operatorname{div}_{M} \operatorname{grad}_{M} f=g^{i j} \frac{\partial^{2}}{\partial x_{i} \partial x_{j}} f+H v_{i} \frac{\partial}{\partial x_{i}} f .
\end{aligned}
$$

By decomposing $Y$ into components normal and tangential to $M, Y=Y^{\perp}+Y^{T}$, we obtain

$$
\operatorname{div}_{M} Y=\operatorname{div}_{M} Y^{T}-\langle H v, Y\rangle .
$$

The induced volume form on $M$ is $d A=v d x$, where $d x$ is Lebesgue measure on $\mathbb{R}^{n}$. Now suppose $G \subset M$ has $C^{1}$ boundary $\partial G$ with outer normal $\sigma$ in $M$. Using (2.6) and Stokes' theorem, we have

$$
\int_{G} \operatorname{div}_{M} Y d A=-\int_{G}\langle H \nu, Y\rangle d A+\int_{\partial G}\langle Y, \sigma\rangle d \mu,
$$

where $d \mu$ is surface measure on $\partial G$. Setting $Y=f \delta g$ in (2.7), where $f, g \in C^{2}(G)$, yields

$$
\begin{aligned}
\int_{G}\left(\langle\delta f, \delta g\rangle+f \Delta_{M} g\right) d A & =\int_{\partial G} f\langle\delta g, \sigma\rangle d \mu, \\
\int_{G}\left(g \Delta_{M} f-f \Delta_{M} g\right) d A & =\int_{\partial G}\langle g \delta f-f \delta g, \sigma\rangle d \mu,
\end{aligned}
$$

where the second result is just Green's formula. Setting $Y=f g e_{\alpha}, f \in C_{c}^{1}(M)$, $g \in C^{1}(M)$ gives

$$
\begin{aligned}
\int_{M} f \delta_{n+1} g d A & =-\int_{M} g \delta_{n+1} f d A-\int_{M} \frac{1}{v} f g H d A, \\
\int_{M} f \delta_{i} g d A & =-\int_{M} g \delta_{i} f d A-\int_{M} v_{i} f g H d A, \quad i=1, \ldots, n .
\end{aligned}
$$

The restriction of the Lorentz distance function to $M$ is very important. By abuse of notation, we treat $x, y \in \mathbb{R}^{n}$ also as points of $M$, and define

$$
l(x, y)=\left(|x-y|^{2}-(u(x)-u(y))^{2}\right)^{1 / 2} .
$$

The distance from a fixed centre point $(\xi, u(\xi))$ will be written $l(x)$ if there is no chance of confusion. Direct calculation using (2.5) then gives

$$
\begin{aligned}
\|\delta l\|^{2} & =1+l^{-2}\langle v, X-\xi\rangle^{2}, \\
\Delta_{M}\left(\frac{1}{2} l^{2}\right) & =n+\langle H v, X-\xi\rangle,
\end{aligned}
$$

where $X-\xi$ is the vector $(x-\xi, u(x)-u(\xi)) \in \mathbb{L}^{n+1}$, and $l=l(x)$ is taken relative to $(\xi, u(\xi))$. The Lorentz ball is then

$$
L_{R}=L_{R}(\xi)=\{(x, u(x)) \in M ; l(x, \xi)<R\} \subset M,
$$


and has projection $K_{R}$ into $\mathbb{R}^{n}$,

$$
K_{R}=K_{R}(\xi)=\left\{x \in \mathbb{R}^{n}, l(x, \xi)<R\right\} .
$$

Since $|D u|<1,(x-\xi) \cdot D\left(\frac{1}{2} l^{2}\right)>|x-\xi|^{2}-|u(x)-u(\xi)| \cdot|x-\xi|$, so $l$ is increasing on outward rays from $\xi$, and $K_{R}$ is starlike with respect to $\xi$. In case $u$ is defined only over a (nonconvex) domain $\Omega$, so $u$ is perhaps not acausal, we must modify this definition to ensure that $K_{R}$ is still starlike. Thus in general we define $K_{R}$ by

$$
K_{R}(\xi)=\{x \in \Omega ; \overline{x \xi} \subset \Omega, l(x, \xi)<R\} \subset \Omega
$$

where $\overline{x \xi}$ is the line segment joining $x$ and $\xi$. This agrees with the previous definition when $K_{R} \subset \subset \Omega$.

We can now demonstrate monotonicity and mean-value formulae analogous to those in [MS]. For convenience, take the fixed centre point $(\xi, u(\xi))=(0,0)$, and let $X$ denote the position vector $(x-\xi, u(x)-u(\xi)) \in \mathbb{L}^{n+1}$. Let $f \in C^{2}(M)$, and substitute $f$ and $g=\frac{1}{2}\left(\varrho^{2}-l^{2}\right)$, where $\varrho$ is a constant, into Green's formula (2.8),

$$
\int_{G}\left(\frac{1}{2}\left(\varrho^{2}-l^{2}\right) \Delta_{M} f+f \Delta_{M}\left(\frac{1}{2} l^{2}\right)\right) d A=\int_{\partial G}\left\langle\frac{1}{2}\left(\varrho^{2}-l^{2}\right) \delta f+f \delta\left(\frac{1}{2} l^{2}\right), \sigma\right\rangle d \mu .
$$

Now let $G=L_{\varrho}$, and assume $\varrho>0$ is such that $\partial L_{\varrho} \cap \partial M=\emptyset$, so $\sigma=\|\delta l\|^{-1} \delta l$. Using (2.10), this becomes

$$
\int_{L_{Q}}\left\{n f+\frac{1}{2}\left(\varrho^{2}-l^{2}\right) \Delta_{M} f+f H\langle X, v\rangle\right\} d A=\int_{\partial L_{\varrho}} f l\|\delta l\| d \mu .
$$

Using the following special case of Federer's coarea formula [F, Theorem 3.2.12],

$$
D_{\varrho}\left\{\int_{L_{\varrho}} f d A\right\}=\int_{\partial L_{\varrho}} f\|\delta l\|^{-1} d \mu
$$

and (2.10), we arrive at the monotonicity formula

$$
\begin{aligned}
D_{\varrho}\left\{\varrho^{-n} \int_{L_{\varrho}} f d A\right\}= & \int_{L_{\varrho}} \varrho^{-n-1}\left(\frac{1}{2}\left(\varrho^{2}-l^{2}\right) \Delta_{M} f+f H\langle X, v\rangle\right) d A \\
& -D_{\varrho}\left\{\int_{L_{\varrho}} f l^{-n-2}\langle X, v\rangle^{2} d A\right\} .
\end{aligned}
$$

Note that the integral in the final term tends to 0 as $\varrho \downarrow 0$, since $M$ is $C^{2}$ and strictly spacelike, and thus $\langle X, v\rangle=O\left(|x|^{2}\right)$ as $|x| \rightarrow 0$. Also, since $l$ approximates geodesic distance in $M$ for $|x-\xi|$ small, if $f$ is continuous at $\xi$, we have

$$
\varrho^{-n} \int_{L_{Q}(\xi)} f d A \rightarrow \omega_{n} f(\xi) \text { as } \varrho \downarrow 0,
$$

where $\omega_{n}$ is the volume of the unit ball in $\mathbb{R}^{n}$.

We will apply (2.15) with $f=v^{\alpha}, \alpha>0$ to obtain important estimates. By direct calculation, using $D_{j} v_{i}=v^{-1} g^{i k} u_{k j}$ and (2.3)-(2.5),

$$
\begin{aligned}
\|\delta v\|^{2} & =g^{i j} v_{k} v_{l} u_{i k} u_{j l}, \\
\Delta_{M} v & =-v\|A\|^{2}+\frac{2}{v}\|\delta v\|^{2}-v^{2} \delta_{n+1} H .
\end{aligned}
$$


This formula can also be derived from the Jacobi field equation. Using (2.3), (2.5), and (2.16) we now have

$$
\begin{aligned}
\Delta_{M} v^{\alpha}= & \alpha v^{\alpha-1} \Delta_{M} v+\alpha(\alpha-1) v^{\alpha-2}\|\delta v\|^{2} \\
= & -\alpha v^{\alpha-2}\left\{\sum_{i, j=1}^{n} u_{i j}^{2}-\alpha\left(u_{i i}\right)^{2}-(1-\alpha) v H u_{i i}+v^{2} H^{2}+(1-\alpha)\left|v_{i} u_{i j}\right|^{2}\right\} \\
& -\alpha \delta_{n+1}\left(v^{\alpha+1} H\right),
\end{aligned}
$$

where $\left|v_{i} u_{i j}\right|^{2}=\sum_{j=1}^{n}\left(\sum_{i=1}^{n} v_{i} u_{i j}\right)^{2}$. Using the inequalities $a b \leqq \varepsilon a^{2}+\frac{1}{4 \varepsilon} b^{2}$ for any $\varepsilon>0$,

we can estimate

$$
\left(u_{i i}\right)^{2} \leqq n \sum_{1}^{n} u_{i i}^{2} \leqq n \sum u_{i j}^{2}
$$

$$
\Delta_{M} v^{\alpha} \leqq-C v^{\alpha-2}\left(\sum u_{i j}^{2}+\left|v_{i} u_{i j}\right|^{2}\right)+\frac{1}{4} v^{\alpha} H^{2}-\alpha \delta_{n+1}\left(v^{\alpha+1} H\right),
$$

where $\alpha<\frac{1}{n}$ and $C$ are positive constants depending only on $n$.

Using this estimate in the monotonicity formula (2.15) yields

$$
\begin{aligned}
& D_{\varrho}\left\{\varrho^{-n} \int_{L_{\varrho}} v^{\alpha} d A\right\} \\
& \leqq-C \int_{L_{\varrho}} \frac{1}{2} \varrho^{-n-1}\left(\varrho^{2}-l^{2}\right)\left(\sum_{i, j=1}^{n} u_{i j}^{2}+\sum_{j=1}^{n}\left|v_{i} u_{i j}\right|^{2}\right) v^{\alpha-2} d A \\
&+\int_{L_{e}} \frac{1}{2} \varrho^{-n-1}\left(\varrho^{2}-l^{2}\right)\left(\frac{1}{4} v^{\alpha} H^{2}-\alpha \delta_{n+1}\left(v^{\alpha+1} H\right)\right) d A \\
&+\int_{L_{\varrho}} \varrho^{-n-1} H\langle X, v\rangle v^{\alpha} d A-D_{\varrho}\left\{\int_{L_{e}}\langle X, v\rangle^{2} l^{-n-2} v^{\alpha} d A\right\} .
\end{aligned}
$$

If $\zeta \in C^{1}(\mathscr{U}), M \subset \mathscr{U} \subset \mathbb{L}^{n+1}$, and $D_{n+1} \zeta=0$, then

$$
\|\delta \zeta\|^{2}=g^{i j} D_{i} \zeta D_{j} \zeta=|D \zeta|^{2}+\left(v_{i} D_{i} \zeta\right)^{2} .
$$

Since $v \delta_{n+1} \zeta=v_{i} D_{i} \zeta$ by (2.5), we thus have

$$
\left|v \delta_{n+1} \zeta\right| \leqq\|\delta \zeta\|, \text { provided } D_{n+1} \zeta=0 .
$$

The $\delta_{n+1}\left(v^{\alpha+1} H\right)$ term in (2.17) may be estimated by integrating by parts (2.9) and using (2.10) and (2.18):

$$
\begin{aligned}
-\int_{L_{\varrho}}\left(\varrho^{2}-l^{2}\right) \delta_{n+1}\left(v^{\alpha+1} H\right) d A & =\int_{L_{\varrho}}\left(\varrho^{2}-l^{2}\right) v^{\alpha} H^{2} d A-2 \int_{L_{\varrho}} v^{\alpha+1} H l \delta_{n+1} l d A \\
& \leqq \int_{L_{\varrho}} \varrho\left(R^{-1}\|\delta l\|^{2}+(\varrho+R) H^{2}\right) v^{\alpha} d A,
\end{aligned}
$$

where $R \geqq \varrho$ will be determined later.

Now suppose $\sup |H| \leqq \Lambda<\infty$, and estimate $H\langle X, v\rangle \leqq \frac{1}{2} l^{2} H^{2}+\frac{1}{2} l^{-2}\langle X, v\rangle^{2}$. Defining

$$
\psi(\varrho)=\varrho^{-n} \int_{L_{\varrho}} v^{\alpha} d A
$$


the monotonicity formula now becomes

$$
\begin{aligned}
\psi^{\prime}(\varrho) & -\left(\Lambda^{2} \varrho+\frac{1}{2} \alpha R^{-1}+\frac{1}{2} \alpha \Lambda^{2} R\right) \psi(\varrho) \\
\leqq & -C \varrho^{-n-1} \int_{L_{\varrho}} \frac{1}{2}\left(\varrho^{2}-l^{2}\right)\left(\sum u_{i j}^{2}+\left|v_{i} u_{i j}\right|^{2}\right) v^{\alpha-2} d A \\
& +\varrho^{-n-1} \int_{L_{\varrho}}\langle X, v\rangle^{2} l^{-2} v^{\alpha} d A-D_{\varrho}\left\{\int_{L_{\varrho}}\langle X, v\rangle^{2} l^{-n-2} v^{\alpha} d A\right\} .
\end{aligned}
$$

For any $f \in C^{1}(\mathbb{R})$, the coarea formula (2.14) shows that

$$
f^{\prime}(\varrho) \int_{L_{\varrho}} g d A=D_{\varrho}\left\{\int_{L_{\varrho}}(f(\varrho)-f(l)) g d A\right\},
$$

and hence we can write the last two terms of $(2.19)$ as $-T^{\prime}(\varrho)$, where

$$
T(\varrho)=\int_{L_{\varrho}}\left[\left(1-\frac{1}{n}\right) l^{-n-2}+\frac{1}{n} \varrho^{-n} l^{-2}\right]\langle X, v\rangle^{2} v^{\alpha} d A .
$$

Using the integrating factor

$$
I(\varrho)=\exp \left\{-\frac{1}{2}\left(\Lambda^{2} \varrho^{2}+\alpha R^{-1} \varrho+\alpha \Lambda^{2} R \varrho\right)\right\},
$$

(2.19) becomes

$$
(I(\varrho) \psi(\varrho))^{\prime} \leqq-C I(\varrho) \varrho^{-n-1} \int_{L_{\varrho}} \frac{1}{2}\left(\varrho^{2}-l^{2}\right)\left(\sum u_{i j}^{2}+\left|v_{i} u_{i j}\right|^{2}\right) v^{\alpha-2} d A-I(\varrho) T^{\prime}(\varrho) .
$$

To help integrate this expression, define the step function $\lambda(t)=0, t<0$ and $\lambda(t)=1$, $t \geqq 0$. Then for any $g$ integrable on $M$, we can write

$$
\int_{L_{\varrho}} g d A=\int_{M} \lambda(\varrho-l) g d A
$$

Now integrating (2.20) from 0 to $R$ and using this device and Fubini's theorem gives

$$
\begin{aligned}
I(R) \psi(R)-\omega_{n} v^{\alpha}(\xi) \leqq & -C \int_{L_{R}}\left(\sum u_{i j}^{2}+\left|v_{i} u_{i j}\right|^{2}\right) v^{\alpha-2} \\
& \cdot\left(\int_{0}^{R} \frac{1}{2} \varrho^{-n-1}\left(\varrho^{2}-l^{2}\right) I(\varrho) \lambda(\varrho-l) d \varrho\right) d A-\int_{0}^{R} I(\varrho) T^{\prime}(\varrho) d \varrho .
\end{aligned}
$$

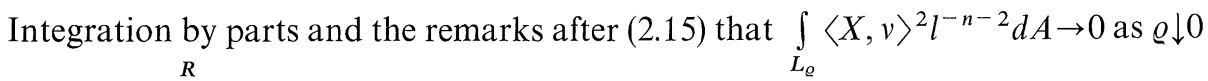
shows that $\int_{0}^{R} I(\varrho) T^{\prime}(\varrho) d \varrho \geqq 0$ and may be discarded, yielding

$$
\omega_{n} v^{\alpha}(\xi) \geqq I(R) R^{-n} \int_{L_{R}} v^{\alpha} d A+C I(R) \int_{L_{R}} S_{R}(l)\left(\sum u_{i j}^{2}+\left|v_{i} u_{i j}\right|^{2}\right) v^{\alpha-2} d A,
$$

where

$$
S_{R}(l)= \begin{cases}\frac{1}{n(n-2)} l^{2-n}+\frac{1}{2 n} l^{2} R^{-n}-\frac{1}{2(n-2)} R^{2-n}, & n \geqq 3, \\ \frac{1}{2} \log (R / l)-\frac{1}{4}\left(1-l^{2} / R^{2}\right), & n=2,\end{cases}
$$

so $S_{R}(l)>0$ for $l<R$.

One estimate which can be derived from (2.21) is 
Lemma 2.1. Let $\Omega$ be a domain in $\mathbb{R}^{n}$ and let $u \in C^{2}(\Omega)$ be strictly spacelike and have bounded mean curvature, $\sup _{\Omega}|\mathscr{M} u| \leqq \Lambda$. Let $\xi \in \Omega$ and $R>0$ be such that $K_{2 R}(\xi) \subset \subset \Omega$, where $K_{\varrho}(\xi)$ is the connected Lorentz ball defined by (2.12). Then there are positive constants $\alpha<\frac{1}{n}$ and $C$ depending only on $n$, such that

$$
C \exp \left(\Lambda^{2} R^{2}+1\right) v^{\alpha}(\xi) \geqq R^{-n} \int_{K_{R}(\xi)} v^{\alpha+1} d x+R^{2-n} \int_{K_{R}(\xi)} \sum u_{i j}^{2} d x
$$

Proof. Note that for $l<R / 2, S_{R}(l)>C(n) R^{2-n}$. By approximation, (2.21) holds for $u \in C^{2}(\Omega)$ also. Then using the definition of $I(R)$ and discarding parts of $(2.21)$, we arrive at (2.22).

\section{Barrier Functions and Global Gradient Estimates}

An important property of the Lorentz mean curvature operator $\mathscr{M}$ is that there are spherically symmetric functions defined on $\mathbb{R}^{n}-\{0\}$ with constant mean curvature. The geometry of these functions is such that they prove very useful as barrier functions. Two important applications are the "contained light ray" Theorem 3.2 (cf. [MT, p. 124]) and the boundary gradient estimate Corollary 3.4, which leads to the solvability of the Dirichlet problem for sufficiently smooth mean curvature, boundary and data.

Suppose $w$ is spherically symmetric about $\xi \in \mathbb{R}^{n}$, so $w=w(r)$, where $r=|x-\xi|$. Let ' denote differentiation with respect to $r$, so

$$
\mathscr{M}(w)=r^{1-n}\left(\frac{r^{n-1} w^{\prime}(r)}{\sqrt{1-w^{\prime}(r)^{2}}}\right)^{\prime} .
$$

Thus the equation $\mathscr{M}(w)=\Lambda$, constant, may be integrated giving

$$
w(x)=w(\xi)+\int_{0}^{|x-\xi|} \frac{K+\frac{1}{n} \Lambda t^{n}}{\sqrt{t^{2 n-2}+\left(K+\frac{1}{n} \Lambda t^{n}\right)^{2}}} d t,
$$

where $K$ and $w(\xi)$ are constants of integration. Two properties of $w$ are immediate from (3.1):

(i) $|D w|<1$ except at $\xi$, where $w$ has a light-cone-like singularity (unless $K=0$, when $w$ is a hyperboloid). Also, $(1-|D w|)$ is $O\left(|x-\xi|^{2 n-1}\right)$, positive near $\xi$.

(ii) By taking $K$ sufficiently large positive (negative), $w$ can be made arbitrarily close to the forward (backward) light cone from $\xi$ over a bounded set.

We note that the analogous computation for the Euclidean mean curvature yields solutions defined only in the complement of a ball of strictly positive radius. Although such solutions can be useful, in this case the solutions (3.1) provide much 
stronger information. For $K>0$ and $\Lambda \in \mathbb{R}$ we have candidate barrier functions

$$
w^{ \pm}(x)=w^{ \pm}(\xi) \pm \int_{0}^{|x-\xi|} \frac{K-\frac{1}{n} \Lambda t^{n}}{\sqrt{t^{2 n-2}+\left(K-\frac{1}{n} \Lambda t^{n}\right)^{2}}} d t
$$

with mean curvatures $\mp \Lambda$. Then by noting that $D^{2} w$ is arbitrarily large in transverse radial directions near $\xi$, and then choosing $K$ sufficiently large and $\xi \in \mathbb{R}^{n}$ appropriately, we see geometrically that the functions $w^{ \pm}$do indeed yield barrier functions. This observation is important enough to formulate precisely (the detailed proof is given in the appendix).

Proposition 3.1. Suppose $\partial \Omega \cap \mathscr{U}$ is $C^{2}, \mathscr{U}$ open, $\Gamma \subset \partial \Omega \cap \mathscr{U}$ is compact, and suppose the boundary data $\varphi: \partial \Omega \rightarrow \mathbb{R}$ has an extension $\bar{\varphi}$ satisfying

$$
\begin{aligned}
|\bar{\varphi}(x)-\bar{\varphi}(y)| \leqq\left(1-\theta_{0}\right)|x-y|, \quad x \in \bar{\Omega}, \quad y \in \Gamma, & \\
\sup _{\mathscr{U} \cap \partial \Omega}\left|D^{2} \bar{\varphi}\right| & \leqq \kappa
\end{aligned}
$$

for some constants $\theta_{0}>0, \kappa$. Let $\Lambda \geqq 0$ be given, and $x_{0} \in \Gamma$. Then there is $K>0$, and base points $\left(\xi^{ \pm}, w^{ \pm}\left(\xi^{ \pm}\right)\right)$such that the functions $w^{ \pm}$defined by (3.2) satisfy

$$
\begin{aligned}
& \left.\begin{array}{l}
w^{+}(x)>\varphi(x) \\
w^{-}(x)<\varphi(x)
\end{array}\right\}, \quad x \in \partial \Omega \backslash\left\{x_{0}\right\}, \\
& w^{ \pm}\left(x_{0}\right)=\varphi\left(x_{0}\right) \text {, } \\
& \mathscr{M}\left(w^{ \pm}\right)=\mp \Lambda \text {. }
\end{aligned}
$$

Further, there is a strictly positive constant $\theta=\theta\left(\kappa, \theta_{0}, \Lambda, \Omega, \Gamma, \mathscr{U}\right)$ independent of $x_{0}$ such that

$$
\sup _{\bar{\Omega}}\left|D w^{ \pm}\right| \leqq 1-\theta<1 \text {. }
$$

The final estimate (3.3) shows that for $u \in C^{2}(\Omega)$, such that $|\mathscr{M} u| \leqq \Lambda, u=\varphi$ on $\partial \Omega$, we have the boundary gradient estimate $|D u(x)| \leqq 1-\theta, x \in \Gamma$, provided $D u(x)$ exists. Note also that this assumes that $\bar{\varphi}$ is (strictly) acausal - this requirement will later be weakened to $\bar{\varphi}$ strictly spacelike.

The next result shows if graph $u$ contains a segment of light ray, then it contains the whole of the ray extended to $\partial \Omega \times \mathbb{R}$.

Theorem 3.2. Let $u \in C^{0,1}(\Omega)$ be a solution to the variational problem (1.3) with bounded $H$. Suppose there is a line segment $\overline{x_{0} x_{1}} \subset \subset \Omega$ such that

$$
u\left(x_{t}\right)=u\left(x_{0}\right)+t\left|x_{0}-x_{1}\right|, \quad \forall t \in[0,1],
$$

where $x_{t}=x_{0}+t\left(x_{1}-x_{0}\right)$. Then this equation holds for all $t \in \mathbb{R}$ such that $x_{t} \in \Omega$ and $\overline{x_{0} x_{t}} \subset \Omega$.

Proof. Suppose (3.4) does not hold for $t<0$ [a completely analogous argument holds if (3.4) fails for $t>1]$. This means

$$
u\left(x_{t}\right)>u\left(x_{0}\right)+t\left|x_{0}-x_{1}\right|, \text { for some } t<0 .
$$


By choosing a new $x_{0}$ and $x_{1}$ (on the line containing the original segment), we can assume (3.4) $\forall t \in\left[-\frac{1}{4}, 1\right]$, (3.5) with $t=-\frac{1}{2}$, and that the Euclidean ball $B_{1}=B_{2\left|x_{0}-x_{1}\right|}\left(x_{1}\right) \subset \subset \Omega$. Let $B_{0}=B_{\frac{1}{2}\left|x_{0}-x_{1}\right|}\left(x_{0}\right)$, and let $C_{1}$ and $C_{2}$ denote the backward light cones with apexes at $\left(x_{1}, u\left(x_{1}\right)\right)$ and $\left(x_{0}, u\left(x_{0}\right)\right)$ respectively. Then for $x \in B_{0} \backslash\left\{x_{t}: t \in\left[-\frac{1}{2}, 0\right]\right\}$, we have

$$
u(x) \geqq C_{1}(x)>C_{2}(x) .
$$

We can now use the strict inequality (3.5) for $u\left(x_{t}\right), t=-\frac{1}{2}$. Thus

$$
u(x)>C_{2}(x), \quad \forall x \in \partial B_{0},
$$

and we can find $K$ sufficiently large such that the spherically symmetric function $w^{-}(x)$ with mean curvature $\Lambda=\sup H$ and base point $\left(x_{0}, u\left(x_{0}\right)\right)$ satisfies

$$
u(x)>w^{-}(x)>C_{2}(x), \quad \forall x \in \partial B_{0} .
$$

But $w^{-}$is strictly spacelike away from $x_{0}$, so $w^{-}\left(x_{t}\right)>u\left(x_{t}\right), t \in\left[-\frac{1}{4}, 0\right)$, and this contradicts the comparison Lemma 1.2 applied with $\Omega=B_{0} \sim\left\{x_{0}\right\}$.

The following lemma will be used to prove an a priori boundary gradient bound for strictly spacelike data, generalizing Proposition 3.1.

Lemma 3.3. Let $\Omega \subset \mathbb{R}^{n}$ be a bounded domain and $\Lambda$ be a given constant. Define the function classes:

$$
\mathscr{D} \subset\left\{\psi \in C^{0}(\partial \Omega):|\psi(x)-\psi(y)|<|x-y| \text { whenever } x \neq y \text { and } \overline{x y} \subset \Omega\right\}
$$

and $\mathscr{D}$ is compact with respect to the uniform norm on $C^{0}(\partial \Omega)$,

$$
\mathscr{F}=\left\{u \in C^{2}(\Omega):|D u(x)|<1, \forall x \in \Omega,|\mathscr{M} u| \leqq \Lambda \text { and } u=\psi \text { on } \partial \Omega \text { for some } \psi \in \mathscr{D}\right\} .
$$

Let $r>0$ be given, and let $x_{1}, x_{2} \in \bar{\Omega}$ be any two points satisfying $\left|x_{1}-x_{2}\right|=r$, and the open line segment $\overline{x_{1} x_{2}} \subset \Omega$. Then there is a strictly positive constant $\theta=\theta(n, \Lambda, \Omega, r, \mathscr{D})$ such that, for any $u \in \mathscr{F},\left|u\left(x_{1}\right)-u\left(x_{2}\right)\right| \leqq(1-\theta) r$.

Proof. Suppose there is no such $\theta$. Then we can find a sequence $\left\{u^{k}\right\} \subset \mathscr{F}$, and weakly spacelike $\tilde{u}$ on $\Omega, \tilde{H} \in L^{2}(\Omega)$ such that $\sup _{\Omega}\left|u^{k}-\tilde{u}\right| \rightarrow 0, \mathscr{M}\left(u^{k}\right)=H^{k} \rightarrow \tilde{H}$ in $L^{2}(\Omega)$ and there are points $x_{1}, x_{2} \in \bar{\Omega}$ satisfying $\overline{x_{1} \bar{x}_{2}} \subset \bar{\Omega}$ and $\left|\tilde{u}\left(x_{1}\right)-\tilde{u}\left(x_{2}\right)\right|$ $=\left|x_{1}-x_{2}\right|=r$. But Lemma 1.3 shows that $\tilde{u}$ is a solution of the variational problem with mean curvature $\tilde{H}$ with respect to its boundary values. Theorem 3.2 now shows that the segment of light ray over $\overline{x_{1} x_{2}}$ in graph $u$ extends to a segment of light ray between points of $\partial \Omega \times \mathbb{R}$, contradicting the fact that $\left.\tilde{u}\right|_{\partial \Omega} \in \mathscr{D}$.

Note that this does not give an interior gradient bound.

Corollary 3.4. Let $\Omega \subset \mathbb{R}^{n}$ be a bounded, $C^{2}$ domain, and suppose the boundary data $\varphi$ has an extension $\bar{\varphi} \in C^{2}(\bar{\Omega})$ such that $\sup _{\bar{\Omega}}|D \bar{\varphi}| \leqq 1-\theta_{0}<1, \sup _{\bar{\Omega}}\left|D^{2} \bar{\varphi}\right| \leqq \kappa$ for some constants $\theta_{0}>0$ and $\kappa$. Let $u \in C^{2}(\Omega) \cap C^{1}(\bar{\Omega})$ be strictly spacelike and satisfy $\sup _{\Omega}|\mathscr{M} u| \leqq \Lambda$, and $u=\varphi$ on $\partial \Omega$. Then there is a positive constant $\theta=\theta\left(\theta_{0}, \Lambda, \kappa, \Omega, n\right)$ such that $\sup _{\partial \Omega}|D u| \leqq 1-\theta<1$.

Proof. Let $x_{0} \in \partial \Omega$. Since $\Omega$ is $C^{2},|D \bar{\varphi}| \leqq 1-\theta_{0}$ and $u \in C^{0,1}(\bar{\Omega})$, it follows from Lemma 3.3 that there is $r=r\left(\Omega, \theta_{0}\right)>0$ and $\theta_{1}=\theta_{1}\left(\theta_{0}, \Lambda, \Omega, r, n\right)>0$ such that

$$
\left|u\left(x_{0}\right)-u(x)\right| \leqq\left(1-\theta_{1}\right) r, \quad \forall x \in \Omega \cap \partial B_{r}\left(x_{0}\right) .
$$


Proposition 3.1 applied to $u$ restricted to $B_{r}\left(x_{0}\right)$ yields barrier functions $w^{ \pm}$at $x_{0}$ with $\left|D w^{ \pm}\left(x_{0}\right)\right| \leqq 1-\theta<1$, where $\theta=\theta\left(\theta_{1}, \Lambda, \kappa, \Omega, r, n\right)>0$. This holds uniformly for $x_{0} \in \partial \Omega$, so the result follows.

Using the Jacobi equation (2.16),

$$
\Delta_{M} v_{n+1}=v_{n+1}\|A\|^{2}+\delta_{n+1} H,
$$

we see that when $H$ is constant, $v_{n+1}$ satisfies a maximum principle, and hence Corollary 3.4 yields a global a priori estimate for $|D u|$ and thus the ellipticity of $\mathscr{M}$. However, we can do better:

Theorem 3.5. Let $\Omega \subset \mathbb{R}^{n}$ be a bounded domain, and suppose $u \in C^{2}(\Omega) \cap C^{1}(\bar{\Omega})$ is strictly spacelike and $\sup _{\Omega}|\mathscr{M} u| \leqq \Lambda$. Further suppose $\varphi=\left.u\right|_{\partial \Omega}$ is bounded, and that there is a boundary gradient estimate for $|D u|$ of the form

$$
\sup _{\partial \Omega} v_{n+1}<K, \text { where } v_{n+1}=\frac{1}{\sqrt{1-|D u|^{2}}} .
$$

Then $|D u|$ satisfies the global estimate: $\sup _{\Omega} v_{n+1} \leqq c K\left((L \Lambda)^{n+2}+1\right)$, where $c=c(n)$ and $L=\operatorname{diam} \Omega+\sup _{\partial \Omega}|\varphi|$.

Proof. Note that $c$ will be used to denote any constant depending only on $n$. We may assume $K \geqq 2$. From (2.9), if $\zeta \in C_{c}^{1}(M)$, then

$$
\int_{M} \delta_{n+1} \zeta d A=-\int_{M} v_{n+1} H \zeta d A .
$$

Substituting $\zeta=u\left(v_{n+1}-K\right)_{+}^{q}, q \geqq 2$ and noting that $\delta_{n+1} u=v_{n+1}^{2}-1 \geqq\left(1-K^{-2}\right) v_{n+1}^{2}$ when $v_{n+1} \geqq K$, we have

$$
\begin{aligned}
& \left(1-K^{-2}\right) \int_{M}\left(v_{n+1}-K\right)_{+}^{q} v_{n+1}^{2} d A \\
& \quad \leqq L \Lambda \int_{M}\left(v_{n+1}-K\right)_{+}^{q} v_{n+1} d A+q L \int_{M}\left(v_{n+1}-K\right)_{+}^{q-1}\left|\delta_{n+1} v_{n+1}\right| d A .
\end{aligned}
$$

Using the inequality (2.18) and $K \geqq 2$, this becomes

$$
\begin{aligned}
& \frac{1}{4} \int_{M}\left(v_{n+1}-K\right)_{+}^{q} v_{n+1}^{2} d A \\
& \quad \leqq L^{2} \Lambda^{2} \int_{M}\left(v_{n+1}-K\right)_{+}^{q} d A+q^{2} L^{2} \int_{M}\left(v_{n+1}-K\right)_{+}^{q-2}\left\|\delta v_{n+1}\right\|^{2} d A .
\end{aligned}
$$

We estimate the $\left\|\delta v_{n+1}\right\|^{2}$ integral by noting that (2.8), (2.9), and the Jacobi equation (3.6) give

$$
\int_{M}\left\langle\delta v_{n+1}, \delta \zeta\right\rangle d A \leqq \int_{M}\left(H \delta_{n+1} \zeta+v_{n+1} H^{2} \zeta\right) d A, \quad \zeta \in C_{c}^{1}(M), \quad \zeta \geqq 0 .
$$

Setting $\zeta=\left(v_{n+1}-K\right)_{+}^{q-1}$ yields

$$
\begin{aligned}
& \int_{M}\left(v_{n+1}-K\right)_{+}^{q-2}\left\|\delta v_{n+1}\right\|^{2} d A \\
& \quad \leqq \Lambda \int_{M}\left(v_{n+1}-K\right)_{+}^{q-2} v_{n+1}\left\|\delta v_{n+1}\right\| d A+\frac{1}{q-1} \Lambda^{2} \int_{M}\left(v_{n+1}-K\right)_{+}^{q-1} v_{n+1} d A,
\end{aligned}
$$


and hence, since $q \geqq 2$,

$$
\int_{M}\left(v_{n+1}-K\right)_{+}^{q-2}\left\|\delta v_{n+1}\right\|^{2} d A \leqq 3 \Lambda^{2} \int_{M}\left(v_{n+1}-K\right)_{+}^{q-2} v_{n+1}^{2} d A .
$$

Using this to simplify (3.8), we have

$$
\int_{M}\left(v_{n+1}-K\right)_{+}^{q} v_{n+1}^{2} d A \leqq 13 q^{2} L^{2} \Lambda^{2} \int_{M}\left(v_{n+1}-K\right)_{+}^{q-2} v_{n+1}^{2} d A .
$$

Setting $\beta=\frac{n}{n-1}$, the Sobolev inequality in $\mathbb{R}^{n}$ implies

$$
\left(\int_{\Omega}\left(v_{n+1}-K\right)_{+}^{q \beta} d x\right)^{1 / \beta} \leqq c q \int_{\Omega}\left(v_{n+1}-K\right)_{+}^{q-1}\left|D v_{n+1}\right| d x, \quad c=c(n) .
$$

Since $\left|D v_{n+1}\right| \leqq\left\|\delta v_{n+1}\right\|$, and $d x=v_{n+1} d A$, this becomes

$$
\begin{aligned}
& \left(\int_{\Omega}\left(v_{n+1}-K\right)_{+}^{q \beta} d x\right)^{1 / \beta} \\
& \quad \leqq \frac{1}{2 \varepsilon} c q \int_{M}\left(v_{n+1}-K\right)_{+}^{q} v_{n+1}^{2} d A+\frac{\varepsilon}{2} c q \int_{M}\left(v_{n+1}-K\right)_{+}^{q-2}\left\|\delta v_{n+1}\right\|^{2} d A .
\end{aligned}
$$

Setting $\varepsilon=L q$ and using (3.9) and (3.10), we have finally

$$
\left(\int_{\Omega}\left(v_{n+1}-K\right)_{+}^{q \beta} d x\right)^{1 / \beta} \leqq c q^{2} L \Lambda^{2} \int_{\Omega}\left(v_{n+1}-K\right)_{+}^{q-2} v_{n+1} d x .
$$

Using the inequality $(a+b)^{1 / \beta} \leqq a^{1 / \beta}+b^{1 / \beta}, a, b \geqq 0$ and (3.11) yields

$$
\left(K^{q \beta} L^{n}+\int_{\Omega}\left(v_{n+1}-K\right)_{+}^{q \beta} d x\right)^{1 / \beta} \leqq L^{n-1} K^{q}+c q^{2} L \Lambda^{2} \int_{\Omega}\left(v_{n+1}-K\right)_{+}^{q-2} v_{n+1} d x
$$

By considering the sets where $v_{n+1} \geqq 2 K$ and $v_{n+1}<2 K$ separately, the right side may be estimated by

Defining $I_{q}$ by

$$
c q^{2}\left(L \Lambda^{2}+L^{-1}\right)\left(K^{q} L^{n}+\int_{\Omega}\left(v_{n+1}-K\right)_{+}^{q} d x\right) .
$$

$$
I_{q}=\left(K^{q} L^{n}+\int_{\Omega}\left(v_{n+1}-K\right)_{+}^{q} d x\right)^{1 / q},
$$

and setting $q=2 \beta^{m-1}, m$ a positive integer, we can write (3.12) as

$$
I_{2 \beta^{m}} \leqq\left(C\left(L \Lambda^{2}+L^{-1}\right)^{\frac{1}{2} \beta}\right)^{\beta^{-m}} \beta^{m \beta^{-m}} I_{2 \beta^{m-1}},
$$

and this holds whenever $m \geqq 1$. Iterating yields

$$
I_{2 \beta^{m}} \leqq\left(C\left(L \Lambda^{2}+L^{-1}\right)^{\frac{1}{2} \beta}\right)^{\Sigma \beta^{-\mu}} \beta^{\sum \beta^{-\mu}} I_{2},
$$

where the sums are taken as $\sum_{\mu=1}^{m}$. Using (3.10), $I_{2}$ can be estimated by

$$
\int_{\Omega}\left(v_{n+1}-K\right)_{+}^{2} d x \leqq c L^{2} \Lambda^{2} \int_{\Omega_{+}} v_{n+1} d x, \quad \text { where } \quad \Omega_{+}=\Omega \cap\left\{v_{n+1} \geqq K\right\} .
$$

Then by noting that $v_{n+1} \leqq \varepsilon+\frac{1}{2} \varepsilon^{-1}\left(K^{2}+\left(v_{n+1}-K\right)_{+}^{2}\right)$, and setting $\varepsilon=c L^{2} \Lambda^{2}$, we have

$$
\int_{\Omega}\left(v_{n+1}-K\right)_{+}^{2} d x \leqq c L^{n} K^{2}\left(L^{4} \Lambda^{4}+1\right),
$$


and hence $I_{2} \leqq c L^{n / 2} K\left(L^{2} \Lambda^{2}+1\right)$. Since $\sum_{1}^{\infty} \beta^{-m}=n-1$ and $\sum_{1}^{\infty} m \beta^{-m}=n^{2}-n,(3.13)$ implies

$$
I_{2 \beta^{m}} \leqq c K\left((L \Lambda)^{n+2}+1\right)
$$

We obtain the required estimate by letting $m \rightarrow \infty$ and noting that

$$
\lim _{q \rightarrow \infty}\left(K^{q}+\int_{\Omega}\left(v_{n+1}-K\right)_{+}^{q} d x\right)^{1 / q}=\max \left\{K, \sup _{\Omega}\left(v_{n+1}-K\right)\right\} .
$$

This a priori global gradient bound leads to the following existence theorem for the Dirichlet problem.

Theorem 3.6. Let $\Omega \subset \mathbb{R}^{n}$ be a bounded, $C^{2, \alpha}$ domain for some $\alpha>0$, and suppose the boundary data $\varphi$ is bounded and has an extension $\bar{\varphi} \in C^{2, \alpha}(\bar{\Omega})$ satisfying $|D \bar{\varphi}| \leqq 1-\theta_{0}$ in $\bar{\Omega}$, for some $\theta_{0}>0$. Let $H \in C^{0, \alpha}(\Omega \times \mathbb{R})$ be bounded, with $\sup _{\Omega \times \mathbb{R}}|H| \leqq \Lambda$. Then the Dirichlet problem $\mathscr{M} u(x)=H(x, u(x))$ in $\Omega, u=\varphi$ on $\partial \Omega$ has a strictly spacelike solution $u \in C^{2, \alpha}(\bar{\Omega})$. Further, there is a positive constant $\theta=\theta\left(\Lambda, \Omega, \theta_{0}, \varphi\right)>0$ such that $|D u| \leqq 1-\theta<1$ in $\Omega$.

Proof. The conditions on $\Omega$ and $\varphi$ ensure that Corollary 3.4 and then Theorem 3.5 can be applied, giving a global gradient estimate which guarantees that $\mathscr{M}$ is $a$ priori uniformly elliptic. [GT, Theorem 12.7] now gives an a priori estimate for $\|u\|_{C^{1, \beta}(\bar{\Omega})}$, for some $\beta>0$, and then [GT, Theorem 10.4] gives the required result.

As previously noted, if $H(x, t)$ is non-decreasing in $t$, then the solution is unique. If $H \in C^{k, \alpha}(\Omega \times \mathbb{R}), k>0$, then by [GT, Theorem 6.17], $u \in C^{k+2, \alpha}(\Omega)$ and if $H \in C^{\infty}$, then $u$ is $C^{\infty}$ also.

\section{Main Results for Dirichlet Problem}

The results of the previous sections combine to prove three theorems about the Dirichlet and variational problems. The main result, Theorem 4.1, gives a necessary and sufficient condition for the regularity of the variational solution, and hence for the Dirichlet problem to be solvable. Corollary 4.2 makes explicit the relationship between the Dirichlet and variational problems, while Theorem 4.3 gives an alternative necessary and sufficient condition for the solvability of Dirichlet problem. As an application of these results, we describe and prove a slight generalization of the existence part of Treibergs' recent classification of constant mean curvature hypersurfaces in $\mathbb{L}^{n+1}[\mathrm{~T}]$.

We emphasize that $\Omega$ is only assumed bounded. Thus, if $\partial \Omega$ has pathology, the boundary values will always be taken in the sense of definition (1.1).

The main point of the proof of Theorem 4.1 is showing that the surface cannot "go null" [MT]. We do this by using the estimate (2.22), applied to $C^{\infty}$ approximating hypersurfaces, to show that going null at a point implies going null in an open neighbourhood, and then Theorem 3.2 applies.

Theorem 4.1. Let $\Omega \subset \mathbb{R}^{n}$ be a bounded domain, and let $H$ be a given bounded function, measurable on $\Omega \times \mathbb{R}$ and continuous in the $\mathbb{R}$-component, with $\sup _{\Omega \times \mathbb{R}}|H| \leqq \Lambda$. Suppose also that $\varphi: \partial \Omega \rightarrow \mathbb{R}$ is bounded. 
Then there is a strictly spacelike $u \in C^{1}(\Omega) \cap W^{2,2}(\Omega)$ satisfying

$$
\begin{aligned}
\mathscr{M} u(x) & =H(x, u(x)) \quad \text { in } \Omega, \\
u & =\varphi \quad \text { on } \partial \Omega(\text { in the sense of }(1.1))
\end{aligned}
$$

if and only if there is a spacelike function $\psi: \Omega \rightarrow \mathbb{R}$ with $\psi=\varphi$ on $\partial \Omega$ (in the sense of (1.1)).

Remark. By standard regularity theory for quasilinear uniformly elliptic equations, we know that $u$ will automatically be of class $C^{k+1}\left(C^{\omega}\right)$ in $\Omega$ if $H$ is of class $C^{k}\left(C^{\omega}\right)$ in $\Omega$.

Proof. By mollification we can construct $C^{\infty}$ approximants $\Omega_{k}, \psi_{k}$ which satisfy

$$
\begin{aligned}
& \Omega_{k} \subset \Omega, \quad \operatorname{dist}\left(\Omega_{k}, \partial \Omega\right) \leqq 1 / k, \\
& \sup _{\bar{\Omega}_{k}}\left|D \psi_{k}\right| \leqq 1-\theta_{k}<1 \quad \text { for some } \theta_{k}>0, \\
& \sup _{\bar{\Omega}_{k}}\left|\psi-\psi_{k}\right| \leqq 1 / k .
\end{aligned}
$$

We also mollify $H$ to get $H_{k} \in C^{\infty}(\Omega \times \mathbb{R})$, and such that $H-H_{k} \rightarrow 0$ in $L^{2}(\Omega \times[-a, a])$ for every $a>0$. Theorem 3.6 now gives a strictly spacelike, $C^{\infty}$ solution $u^{(k)}$ to the Dirichlet problem on $\Omega_{k}$ with $H_{k}$ and $\left.\psi_{k}\right|_{\partial \Omega_{k}}$.

By passing to a subsequence, we can find a weakly spacelike $u \in C^{0,1}(\Omega)$ such that $u^{(k)} \rightrightarrows u$ and $\sup _{\Omega_{k}}\left|u^{(k)}-u\right| \leqq 1 / k$. Since (by construction) $H_{k}\left(x, u^{(k)}(x)\right) \rightarrow H(x, u(x))$ in $L^{2}(\Omega)$, Lemma 1.3 shows that $u$ is the solution of the variational problem with mean curvature $H^{*}(x)=H(x, u(x))$ and with boundary data $\varphi$.

We now consider the limit function $u$. Let $l(x, y)$ be the Lorentz distance function with respect to $u$,

$$
l(x, y)=\sqrt{|x-y|^{2}-(u(x)-u(y))^{2}}, \quad x, y \in \Omega,
$$

and let $K_{R}(x)$ be the projected Lorentz ball defined by (2.12). The corresponding objects with respect to $u^{(k)}$ will be denoted by a superscript, e.g. $l^{(k)}, K_{R}^{(k)}$.

Let $x_{0} \in \Omega$. If there is $y \in \partial \Omega$ such that $l\left(x_{0}, y\right)=0$ and $\overline{x_{0} y} \subset \Omega$, then Theorem 3.2 shows that this segment extends to a light ray between points of $\partial \Omega \times \mathbb{R}$, contradicting the condition that $\psi$ is spacelike. Thus there is $R>0$ such that $K_{4 R}\left(x_{0}\right) \subset \subset \Omega$. Simple estimates of the functions $l, l^{(k)}$ show that there is $\frac{1}{4} R>r>0$ and $k_{1}$ such that for $\left|x-x_{0}\right|<r$ and $k>k_{1}, K_{\frac{1}{4} R}\left(x_{0}\right) \subset K_{\frac{1}{2} R}^{(k)}(x) \subset K_{R}\left(x_{0}\right)$, and also $K_{2 R}^{(k)}(x) \subset \subset \Omega$. Now applying the estimate (2.22) to $u^{(k)}$, using these inclusions and noting that $B_{r}\left(x_{0}\right) \subset K_{r}\left(x_{0}\right)$, we obtain

$$
\begin{gathered}
\int_{K_{\frac{i}{2} R}^{(k)}(x)} \sum u_{i j}^{(k)^{2}} d x \leqq c(n, \Lambda, R)\left(v^{(k)}(x)\right)^{\alpha}, \quad \forall x \in B_{r}=B_{r}\left(x_{0}\right), \\
\int_{B_{r}\left(x_{0}\right)} \sum u_{i j}^{(k)^{2}} d x \leqq c(n, \Lambda, R), \\
\left\|v^{(k)}\right\|_{L^{2}\left(B_{r}\right)} \leqq\left\|v^{(k)}\right\|_{L^{2}\left(K_{\frac{1}{2} R}\left(x_{0}\right)\right)} \leqq c(n, \Lambda, R)\left(v^{(k)}(x)\right)^{\alpha}, \quad \forall x \in B_{r} .
\end{gathered}
$$

The estimate (4.2) shows that $\left\{u^{(k)}\right\}$ is bounded in the Sobolev space $W^{2,2}\left(B_{\mathrm{r}}\right)$, so by Rellich's theorem and the weak compactness of bounded sets in $W^{2,2}$, there 
is a subsequence converging strongly in $W^{1,2}\left(B_{r}\right)$ and weakly in $W^{2,2}\left(B_{r}\right)$. By passing to this subsequence, we see that $u$ has weak second derivatives and

$$
\begin{aligned}
& u^{(k)} \rightarrow u \text { in } \quad W^{1,2}\left(B_{r}\right), \\
& u^{(k)} \rightarrow u \text { in } \quad W^{2,2}\left(B_{r}\right) .
\end{aligned}
$$

Then (4.4) implies that $\left\|v^{(k)}\right\|_{L^{2}\left(B_{r}\right)} \rightarrow\|v\|_{L^{2}\left(B_{r}\right)}$. Now if $\|v\|_{L^{2}\left(B_{r}\right)}=0$, (4.1) and (4.5) show that

$$
\left\|u_{i j}\right\|_{L^{2}\left(B_{r}\right)} \leqq \liminf _{k \rightarrow \infty}\left\|u_{i j}^{(k)}\right\|_{L^{2}\left(B_{r}\right)} \leqq c \liminf _{k \rightarrow \infty}\left(\inf _{B_{r}}\left(v^{(k)}\right)^{\alpha}\right)=0 .
$$

Then $D u$ is constant in $B_{r}$, and since $|D u|=1$ a.e. in $B_{r},\left.u\right|_{B_{r}}$ is linear with slope 1 . In particular, graph $u$ contains a piece of light ray which is impossible, as before. Thus $\|v\|_{L^{2}\left(B_{r}\right)}>0$, and then (4.3) shows that $v^{(k)}(x) \geqq c>0$ for all $x \in B_{r}$ and $k \geqq k_{1}$. Hence

$$
|D u(x)|^{2} \leqq 1-c^{2}<1, \quad \forall x \in B_{r}\left(x_{0}\right) .
$$

Thus $\operatorname{Lip}_{B_{r}} u \leqq \sqrt{1-c^{2}}<1$, so [GT, Sect. 10.5] shows that $u$ is a weak solution of the Dirichlet problem. Then [GT, Theorem 8.24], applied to $D u$ as in Theorem 2.1, shows that for $H$ measurable $D u$ is Hölder continuous in a neighbourhood of $x_{0}$, so $u \in C^{1, \beta}(\Omega) \cap W^{2,2}(\Omega)$ for some $\beta>0$.

Remarks. (i) The local estimate (4.6) guarantees that $u$ is strictly spacelike in $\Omega$. It is easy however to construct examples where this does not extend to $\bar{\Omega}$.

(ii) If $H \in C^{0, \alpha}(\Omega \times \mathbb{R})$, then [GT, Theorem 6.13] shows that $u \in C^{2, \alpha}(\Omega)$, and if $H$ is $C^{k, \alpha}, C^{\infty}$ then $u$ will be $C^{k+2, \alpha}, C^{\infty}$ respectively.

(iii) As observed previously, $u$ will be unique if $H(x, t)$ is non-decreasing in $t$.

If the boundary data $\varphi$ only satisfies a weakly spacelike condition, then the arguments of Theorem 4.1 show that $u$ is regular except along contained light rays. This clarifies the relationship between the Dirichlet and variational problems:

Corollary 4.2. Let $\Omega, H$, and $\varphi$ be as in Theorem 4.1, except that the extension $\psi$ of $\varphi$ need only be weakly spacelike. Define the singular set

$$
K=\{\overline{x y}: x, y \in \partial \Omega, x \neq y, \overline{x y} \subset \Omega \text { and }|\varphi(x)-\varphi(y)|=|x-y|\} .
$$

Then any solution $u$ of the variational problem is strictly spacelike and satisfies $\mathscr{M} u=H$ on $\Omega \backslash K$. Furthermore,

$$
u(t x+(1-t) y)=t \varphi(x)+(1-t) \varphi(y), \quad 0<t<1,
$$

where $x, y \in \partial \Omega$ are such that $|\varphi(x)-\varphi(y)|=|x-y|$, and $\overline{x y} \subset \Omega$.

Proof. Proceed exactly as in Theorem 4.1. If $x_{0} \in \Omega$, then either $l\left(x_{0}, \partial \Omega\right)=0$ and hence $x_{0} \in K$, or the estimates of Sect. 2 apply.

We can express the conditions of the preceding theorems neatly by defining the distance function

$$
d_{\Omega}(x, y)=\inf _{\tau \in \mathscr{P}} \text { length } \tau,
$$

where $\mathscr{P}=\{$ piecewise linear paths in $\Omega$ joining $x$ and $y\}$, and $d_{\Omega}(x, y)=+\infty$ if $\mathscr{P}$ is empty. 
Then it is easy to see that the variational problem (1.3) is solvable iff the boundary data $\varphi$ satisfies

$$
|\varphi(x)-\varphi(y)| \leqq d_{\Omega}(x, y), \quad \forall x, y \in \partial \Omega,
$$

since then the function

$$
\bar{\varphi}(x)=\inf _{y \in \partial \Omega}\left(d_{\Omega}(x, y)+\varphi(y)\right), \quad x \in \Omega,
$$

is a weakly spacelike extension of $\varphi$. If strict inequality holds,

$$
|\varphi(x)-\varphi(y)|<d_{\Omega}(x, y), \quad \forall x, y \in \partial \Omega, \quad x \neq y,
$$

then the singular set of Corollary 4.2 is empty and thus we have shown:

Corollary 4.3. Let $\Omega, H$, and $\varphi$ be as in Theorem 4.1. Then the following conditions are equivalent :

(i) There is a strictly spacelike $u \in C^{1}(\Omega) \cap W^{2,2}(\Omega)$ satisfying the Dirichlet problem.

(ii) The boundary data satisfies

$$
|\varphi(x)-\varphi(y)|<d_{\Omega}(x, y), \quad \forall x, y \in \partial \Omega, \quad x \neq y .
$$

The arguments of Theorem 4.1 show that the problem of constructing surfaces over $\mathbb{R}^{n}$ with prescribed asymptotics and mean curvature reduces to that of constructing suitable barriers at infinity. Many such barriers are given in [T] - we use one of them to illustrate this remark. Treibergs defines the class $\mathscr{Q}$ of positively homogeneous of degree one convex functions on $\mathbb{R}^{n}$ whose gradient has length one wherever defined. He proves the following theorem:

Theorem. Suppose $u$ is an entire, spacelike hypersurface with constant mean curvature $H>0$. Then the blowdown $V_{u}$ of $u$ is in class $\mathscr{Q}$,

$$
V_{u}(x)=\lim _{R \rightarrow \infty} \frac{1}{R} u(R x), \quad x \in \mathbb{R}^{n} .
$$

Conversely, given any function $V \in \mathscr{Q}$, and constant $H>0$, then there is a spacelike hypersurface with mean curvature $H$ such that $V_{u}=V$.

We show that the converse generalizes to non-constant $H$ :

Theorem 4.4. Let the "projective data" $V \in \mathscr{Q}$ be given. Let $H \in C^{0, \alpha}\left(\mathbb{R}^{n}\right)$ be strictly positive, $H(x) \geqq \delta>0$. Then there is a $C^{2, \alpha}$ hypersurface $u$ with $\mathscr{M} u(x)=H(x)$, such that $V_{u}=V$ (i.e. $u$ has projective data $V$ ).

Proof. Let $B_{k}=B_{R_{k}}(0)$ be an exhaustion of $\mathbb{R}^{n}$ by balls. Construct the function

$$
z(x)=\inf _{y \in \mathbb{R}^{n}}\left\{V(y)+\left(n^{2} \delta^{-2}+|x-y|^{2}\right)^{1 / 2}\right\} .
$$

[This construction is due to Treibergs. Note that the term $\left(n^{2} \delta^{-2}+|x-y|^{2}\right)^{1 / 2}$ corresponds to a hyperboloid centred at $y$ with mean curvature $\delta$.] Let $u_{k} \in C^{2}\left(B_{k}\right)$ 
be the solution of the Dirichlet problem over $B_{k}$ with boundary data $\varphi_{k}(x)=V(x)$ $+\frac{1}{2} n \delta^{-1}, x \in \partial B_{k}$. Then the comparison principle shows that $u_{k}(x)<z(x) \leqq V(x)$ $+n \delta^{-1}$ and of course $u_{k}(x)>V(x)$, so we can find a subsequence $u_{k}$ converging uniformly on compact sets. The arguments of Theorem 4.1 then show that the limit function $u$ is $C^{2, \alpha}$ and satisfies $\mathscr{M} u(x)=H(x)$. That $u$ has blowdown $V$, follows from the construction.

We remark that since the asymptotic data is null, the Dirichlet problem over $B_{k}$ has degenerating ellipticity as $k \rightarrow \infty$. Thus regularity of the limit surface cannot be inferred from the usual Schauder estimates [GT].

\section{Appendix. Proof of Proposition 3.1}

Let $D^{\prime}$ denote the tangential gradient operator of $\partial \Omega$ at $x_{0} \in \Gamma$. Assume $x_{0}=0$ and that $e_{n}=(0, \ldots, 0,1)$ is the inward pointing unit normal to $\partial \Omega$ at 0 ; thus $D^{\prime}=\left(D_{1}, \ldots, D_{n-1}, 0\right)$ at 0 . We can also assume that $D^{\prime} \varphi(0)=a e_{1}, e_{1}=(1,0, \ldots, 0)$, $a \in\left[-1+\theta_{0}, 1-\theta_{0}\right]$.

Let $b=b_{\varepsilon} \in \mathbb{R}$ be such that

$$
\frac{1-\left(\Lambda \varepsilon^{n+1} / n\right)}{\sqrt{\varepsilon^{2 n}+\left(1-\left(\Lambda \varepsilon^{n+1} / n\right)\right)^{2}}} \frac{b}{\sqrt{1+b^{2}}}=a
$$

whenever $\varepsilon>0$ is small enough to ensure that such a $b$ exists, and let $w^{+}=w_{\varepsilon}^{+}$be as in (3.2) with $\xi=\xi_{\varepsilon}=\varepsilon(-b, 0, \ldots, 0,-1) / \sqrt{1+b^{2}}$, with $K=\varepsilon^{-1}$ and with $w^{+}(\xi)=0$. The condition (A1) then says precisely that

$$
D^{\prime} w^{+}(0)=a e_{1}=D^{\prime} \varphi(0)
$$

Now let $\eta$ be any unit vector in $\mathbb{R}^{n}$, and let $D_{\eta}$ denote directional differentiation in the direction of $\eta$. Then

$$
D_{\eta} w^{+}(x)=\gamma(|x-\xi|) \frac{(x-\xi)}{|x-\xi|} \cdot \eta
$$

where, for $t \in \mathbb{R}$,

$$
\gamma(t)=\frac{1-\left(\varepsilon \Lambda t^{n} / n\right)}{\sqrt{\varepsilon^{2} t^{2 n-2}+\left(1-\left(\varepsilon \Lambda t^{n} / n\right)\right)^{2}}},
$$

and

$$
D_{\eta} D_{\eta} w^{+}(x) \geqq \frac{\gamma(|x-\xi|)}{|x-\xi|}\left[1-\left(\frac{(x-\xi)}{|x-\xi|} \cdot \eta\right)^{2}\right]-O(\varepsilon)
$$

as $\varepsilon \rightarrow 0$ (uniformly for $x \in \bar{\Omega}$ ).

Next, let $B=\left\{x \in \mathbb{R}^{n}:|x| \leqq \varepsilon^{1 / 2}\right\}$ and note that

$$
\inf _{x \in \bar{\Omega} \sim B} \frac{x-\xi}{|x-\xi|} \cdot \frac{x}{|x|} \geqq 1-O\left(\varepsilon^{1 / 2}\right)
$$


as $\varepsilon \rightarrow 0$. Thus by (A3) we have

$$
\inf _{x \in \bar{\Omega} \sim B} \frac{x}{|x|} \cdot D w^{+}(x) \geqq 1-O\left(\varepsilon^{1 / 2}\right)
$$

as $\varepsilon \rightarrow 0$.

Since $\partial \Omega \cap \mathscr{U}$ is $C^{2}$, we have from (A1) that

$$
\left|\frac{x-\xi}{|x-\xi|} \cdot \frac{x}{|x|}\right| \leqq 1-\left(\theta_{0} / 8\right) \quad \text { for } \quad|x| \leqq \varepsilon \leqq \varepsilon(\Gamma, \mathscr{U}, \Omega), \quad x \in \partial \Omega,
$$

and hence [by (A4)]

$$
\left\langle D^{2} w^{+}(x) \frac{x}{|x|}, \frac{x}{|x|}\right\rangle \geqq \varepsilon^{-1 / 2} \theta_{0} / 8 \text { for }|x| \leqq \varepsilon, \quad x \in \partial \Omega \cap B .
$$

Next consider $x \in \partial \Omega \cap B$ with $|x|>\varepsilon$. For such points we have $(x-\xi) \cdot x \geqq 0$, and hence (A3), (A4) imply [for $\left.\varepsilon \leqq \varepsilon\left(\Gamma, \mathscr{U}, \Omega, \theta_{0}, \Lambda\right)\right]$ that

$$
\text { either } \frac{x}{|x|} \cdot D w^{+}(x) \geqq 1-\left(\theta_{0} / 8\right) \text { or else (A6) holds for } x \text {. }
$$

Since $\left|D^{2} \bar{\varphi}(x)\right| \leqq \kappa$ and $\left|D^{\prime} \varphi(x)\right| \leqq 1-\theta_{0}$ for $x \in \partial \Omega \cap \mathscr{U}$, and since (A2) holds, it now follows directly from (A6) and $\left(\mathrm{A}^{\prime}\right)$,

$$
w^{+}(x)-w^{+}(0)+\varphi(0) \geqq \varphi(x), \quad \forall x \in B \cap \partial \Omega,
$$

provided $\varepsilon>0$ is sufficiently small (depending on $\kappa, \Gamma, \mathscr{U}, \Lambda, \Omega, \theta_{0}$ ), and the inequality is strict for $x \neq x_{0}$.

Finally, (A7) taken together with (A5) and the fact that $|\bar{\varphi}(x)-\bar{\varphi}(y)|$ $\leqq\left(1-\theta_{0}\right)|x-y|, y \in \Gamma, x \in \bar{\Omega}$, now implies

$$
w^{+}(x)-w^{+}(0)+\varphi(0) \geqq \varphi(x), \quad \forall x \in \partial \Omega,
$$

provided $\varepsilon$ is sufficiently small, depending only on $\kappa, \Gamma, \mathscr{U}, \Lambda, \Omega$, and $\theta_{0}$. Then $w^{+}(x)-w^{+}(0)+\varphi(0)$ is the required upper barrier function.

\section{References}

Av Avez, A.: Essais de géométrie Riemannienne hyperbolique globale - applications a la relativité générale. Ann. Inst. Fourier (Grenoble) 13, 105-190 (1963)

B Bancel, D.: Sur le problème de Plateau dans un varété Lorentzienne. C.R. Acad. Sci. Paris 268 A, 403-404 (1978)

BR Bartnik, R.: The Lorentz mean curvature equation. Thesis Melbourne 1980

CE Calabi, E.: Examples of Bernstein problems for some non-linear equations. AMS Symposium on Global Analysis, Berkeley 1968

CY Cheng, S.Y., Yau, S.-T.: Maximal spacelike hypersurfaces in the Lorentz-Minkowski spaces. Ann. Math. 104, 407-419 (1976)

ES Eardley, D., Smarr, L.: Time functions in numerical relativity: marginally bound dust collapse. Phys. Rev. D 19, 2239 (1979)

FH Federer, H.: Geometric measure theory. Berlin, Heidelberg, New York: Springer 1969

FF Flaherty, F.: The boundary value problem for maximal hypersurfaces. P.N.A.S. USA 76, 4765-4767 (1979) 
GT Gilbarg, D., Trudinger, N.S.: Elliptic partial differential equations of second order. Berlin, Heidelberg, New York: Springer 1977

MT Marsden, J., Tipler, F.: Maximal hypersurfaces and foliations of constant mean curvature in general relativity. Phys. Rep. 66, 109-139 (1980)

M Morrey, C.B.: Multiple integrals in the calculus of variations. Berlin, Heidelberg, New York: Springer 1966

MS Michael, J., Simon, L.: Sobolev and mean-value inequalities on generalized submanifolds of $\mathbb{R}^{n}$. Commun. Pure Appl. Math. 26, 361-379 (1973)

$\mathrm{T}$ Treibergs, A.: Entire spacelike hypersurfaces of constant mean curvature in Minkowski space. Invent. Math. 66, 39-56 (1982)

Communicated by S.-T. Yau

Received November 18, 1981; in revised form July 7, 1982 